\title{
EXPLOITING HYPERTEXT VALUATION LINKS FOR BUSINESS DECISION MAKING: A PORTFOLIO MANAGEMENT ILLUSTRATION
}

\author{
P. Balasubramanian \\ Tomas Isakowitz \\ Robert J. Kauffman \\ R. Madhavan
}

Department of Information, Operations, and Management Sciences

Leonard N. Stern School of Business, New York University

44 West $4^{\text {th }}$ Street, New York, NY 10012 


\title{
EXPLOITING HYPERTEXT VALUATION \\ LINKS FOR BUSINESS DECISION MAKING: \\ A PORTFOLIO MANAGEMENT ILLUSTRATION
}

\author{
by \\ P. Balasubramanian \\ Doctoral Program in Information Systems \\ Leonard N. Stern School of Business \\ New York University \\ Tomas Isakowitz \\ Assistant Professor of Information Systems \\ Leonard N. Stern School of Business \\ New York University \\ Robert J. Kauffman \\ Assistant Professor of Information Systems \\ Leonard N. Stern School of Business \\ New York University \\ R. Madhavan \\ Doctoral Program in Information Systems \\ Leonard N. Stern School of Business \\ New York University \\ June 1991 \\ Center for Research on Information Systems \\ Information Systems Department \\ Leonard N. Stern School of Business \\ New York University
}

\section{Working Paper Series}

STERN IS-91-12

The authors wish to express their appreciation to Andrew B. Peake, who was kind enough to agree to participate in the interviews that enabled us to develop the conceptual model used in this paper. All errors are the responsibility of the authors. 
EXPLOITING HYPERTEXT VALUATION LINKS FOR BUSINESS DECISION MAKING:

A PORTFOLIO MANAGEMENT ILLUSTRATION

\begin{abstract}
In this paper we discuss the application of hypertext valuation links to decision support for business problems. Valuation links enable us to relate hypertext link traversal to computation in a way that affects the contents of a hypertext node while retaining the "browsing metaphor" of hypertext. This helps to support quantitative or qualitative reasoning about business problems when described in terms of hypertext nodes that are computational in nature. We illustrate these ideas in the domain of securities analysis and portfolio management, where a "buy side" portfolio manager may need to clarify his understanding of the basis of a "sell siden securities analyst's recommendations about securities that are candidates for inclusion in a portfolio.
\end{abstract}


* maintaining the ability to flexibly switch from subproblem to subproblem as decision making requires;

* $\quad$ solving problems using a more diffuse or non-linear approach, instead of a traditional linear and sequential one.

\subsection{Hypertext in Auditing: An Example}

In fact, the use of hypertext in business settings is now being given increasing consideration. Examples of hypertext applications have now been demonstrated for automotive sales catalogues (Nie90) and auditing (You89, You90). As Nielsen (Nie90) observed:

"Auditing is a natural application for hypertext because it is based on relating information from various sources and checking for consistency. The audit task includes gathering and producing large numbers of documents and linking them together to substantiate the accuracy of the information they contain. A huge amount of information gets distilled into a single financial statement, so links are needed between conclusions and the source data. (Nie90, p. 53)

However, as a whole the functional areas of business have not yet received much attention from hypertext specialists, nor has decision making been addressed to any meaningful extent.

\section{COMPUTING WITH HYPERTEXT}

Next, let's take a closer look at computational hypertext, and the set of concepts that form the basis of our motivation for a new kind of hypertext link that can be applied in business problem solving settings. The term computational hypertext (Nie90) refers to the ability of a hypertext system to perform computations through a programming environment that is integrated into it. Examples of systems that include computational hypertext capabilities are KMS (AMY88), HyperCard and MALUAR (Isa91a).

\subsection{Hypertext Nodes and Hypertext Links: Basics}

The basic building blocks of hypertext applications are nodes and links. Hypertext nodes are the storage locations of chunks of information related to a document, and are alternatively called frames or notecards (AMY88,Hal88). Generally, node types differ according to the storage function that they perform. For example, plain text, a graphical image, an audio track or an executable program can all be stored in nodes. However, some hypertext applications employ just one node type. Hypertext links, on the other hand, represent connections between nodes, based on a relationship that is defined by the system or by the user. Links offer the user a mechanism by which to navigate through a hypertext document, and to discover relationships that are not evident when hypertext is unavailable. The link traversal operation is also referred to as "browsing" in some contexts.

\subsection{Hypertext Concepts}

We now turn to a closer inspection of link types in hypertext. At this time there is no definitive taxonomy for hypertext links, though it might be useful to have one to untangle the varied definitions of hypertext links that occur in the literature. For example, Nielsen (Nie90) differentiates between explicit links and implicit links. (Elsewhere, explicit links have been called extensional links (DeR89) and implicit links have been called virtual structures (Hal88).) Explicit links are static links that have a fixed source and a fixed destination. These are the most common types of links. Implicit links are not explicitly defined; instead, they follow from the properties of the system. An example is the glossary look-up capability in Intermedia. Kibby and Mayes 
traversal. The second type, dynamic links, are automatically traversed whenever some change occurs at the destination which results in a change in the value associated with that node.

The computational power of valuation links is obtained as a result of combining link traversal and node evaluation operations. The result of a change in value of a given node will automatically be propagated via dynamic valuation links to other nodes which in turn might change their value and further propagate change though the dynamic links associated with those nodes and so on until nothing remains to be updated. A specialized propagation algorithm will improve running time while also detecting cycles to avoid infinite loops.

Sidebar 1 below shows an algorithm for hypertext valuation link traversal.

\section{INSERT SIDEBAR 1 ABOUT HERE}

The GUIDE software has no control of that program's output use. Note that this does not involve any propagation through other links as supported by valuation links.

Decision support systems need to provide the user with mechanisms to support cross-referencing of heterogenous information (data, programs, reports, graphs, tables, etc.) as well as tools for performing computations and combining the results of these. For example, a user of a DSS should be able to perform the following sequence of events with ease: extract information from a database, use that information to evaluate certain constraints using a spreadsheet model, solve the constraint problem using a linear program and summarize his conclusions in a report which he creates using a wordprocessor. Furthermore, the paths leading to a particular decision should be documented in the DSS since the system was used as an aid to reach that decision. Traditional hypertext is able to document all textual cross-referencing. When it comes to establishing the connections between the different computational steps however, we need the power of computational hypertext. Furthermore, dynamic valuation links will support the automation of a whole range of procedures involving different processes. For example the sequence of events described above can be automated by establishing dynamic valuation links. Database updates will automatically propagate through the whole chain of processes described there concluding in automatic updates of reports.

Bhargava, Bieber and Kimbrough (BBK88) described a concept called generated value buttons that is similar to valuation links. However, their concept specifies no correlation between link traversal and node content change, nor is the idea of dynamic value changes present. To our knowledge, no specific label has been applied to the kind of hypertext links we describe here, though these fit the computational hypertext concept well. The notion of computational hypertext captures the triggering of program execution via link traversal. Although valuation links can be implemented by programs which affect the contents of the source node, there is a difference between adhoc implementation and the recognition of valuation links as hypertext objects.

\section{ILLUSTRATION DOMAIN: PORTFOLIO MANAGEMENT DECISION SUPPORT}

.We next discuss the background for hypertext support of securities analysis for portfolio management that employs hypertext valuation links. Security analysis is the study of the set of factors that account for variations in the market valuation of a firm's equity. Porfolio management, on the other hand, deals with the process of constructing and managing portfolios of financial instruments so as to maximize return in a world of efficient markets. 
side analysts, specific directives from senior management or the firm's equity committee) and from external sources (indicators about a company, an industry or the market in general; value-added securities analyses from competing firms; and the opinions of independent experts.)

Generally speaking, the buy side and the sell side have the same sources of information at their disposal, though they may have different levels of specific expertise with which to evaluate it. As a result, even if they both had the same assumptions and both used the same analytical models, it is not clear that they would arrive at similar recommendations. In reality, the differences are even greater. In fact, there is a significant asymmetry in the assumptions they employ, the kinds of information to which they have ready access and the basis of their individual decisions, decision basis, and assumptions (Pea91).

This asymmetry gives rise to the potential for conflict in the evaluation of securities which are considered for portfolio investments, as shown in Figure 1 below.

INSERT FIGURE 1 ABOUT HERE

\section{HYPERTEXT VALUATION LINKS FOR SECURITIES ANALYSIS AND PORTFOLIO MANAGEMENT: WORK IN PROGRESS}

We are currently developing an implementation of the conceptual model using the Maluar (Isa91a) system. The example incorporates heuristics for decision making and is to propagate information though valuations links. Figure 2 depicts a preliminary draft of an example.

\section{INSERT FIGURE 2 ABOUT HERE}

Decision support systems need to be able to incorporate elements that will aid a user in the decision making process. These elements include an intuitive user interface and the ability to integrate processes of a different nature. We have argued that via the incorporation of valuation links hypertext provides these tools and therefore represents a desirable platform for such a DSS.

In addition:

* Computational hypertext, especially valuation links, is able to capture complex decision making procedures while assisting the user in their elaboration.

* The portfolio manager example will show how computational hypertext is used as a decision support tool that assists the PM in making decisions.

* This example further shows how valuation links can be used to represent different aspects of reasoning that are present in the securities management environment. 


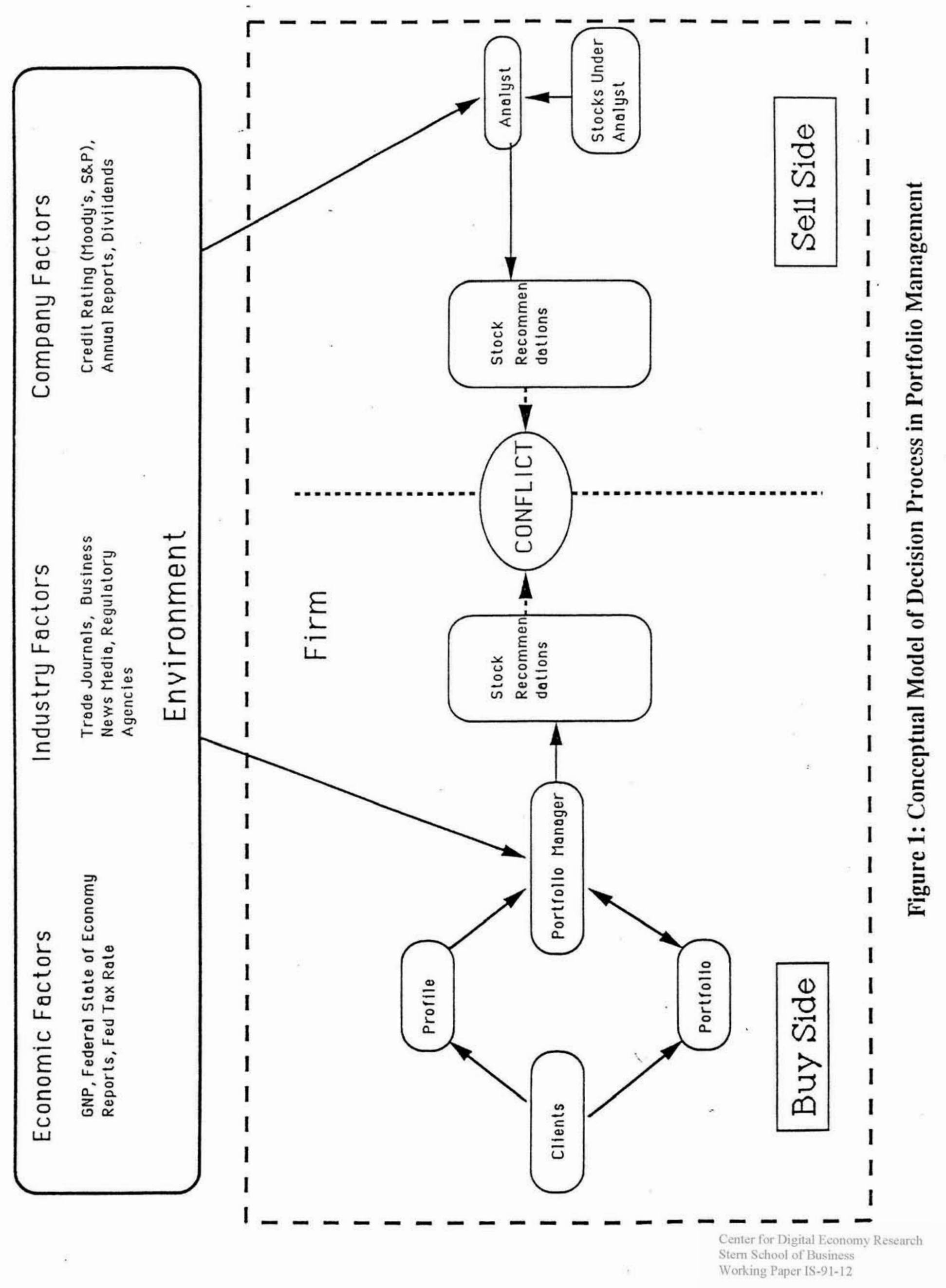




\section{References}

[AK90] F. Afrati and D.C. Koutras. A Hypertext Model Supporting Query Mechanisms. In Proceedings of the European Conference on Hypertext, 1990.

[AM84] R. Acksyn and D. McCracken. ZOG and the USS Carl Vinson: Lessons in Systems Development. In In Proceedings of the First IFIP Conference on Human Computer Interaction., Amsterdam, Netherlands, 1984.

[AMYSS] Robert M. Akscyn, D. L. McCracken, and E.A. Yoder. KMS: A Distributed Hypermedia System for Managing Knowledge in Organizations. Communications of the ACM, 31(7):\$20-\$35, 1988.

[BBKSS] Hemant Bhargava, Michael Bieber, and Steven O. Kimbrough. Oona, Max and the WYWWYWI Principle: Generalyzed Hypertext and Model Management in a Symbolic Programming Environment. In Janice I. DeGross and Margrethe H. Olson, editors, Proceedings of the Ninth ICIS, pages 179-192, 1988.

[Ber75] L.A. Bernstein. In Defense of Fundamental Analysis. Financial Analysis Journal, pages 5i-61, February 1975.

[BK90] Catriel Beeri and Yoram Kornatzky. A Logical Query Language for Hypertext Systems. In Proceedings of the European Conference on Hypertext, 1990.

[Blu71] M.E. Blume. On the Assessment of Risk. Journal of Finance, pages 1-10, March 1971.

[Bye87] T. Byers. Built by Association. PC World, April 1987.

[CG88] B. Campbell and J. M. Goodman. HAM: A general Purpose Hypertext Abstract Machine. Communications of the ACM, July 1988.

[CM89] M. P. Consens and A. O. Mendelzon. Expressing structural Hypertext Queries in GrapLog. In HyperText-89 Proceedings, pages 249-258. ACM, November 5 - 81989. 
[KM89] M. R. Kibby and J. T. Mayes. Towards Intelligent Hypertext. In R. Mc Aleese, editor, HyperText Theory into Practice, pages 164-172. Ablex, 1989.

[Min90] R.P. Minch. Applications and Research Areas for Hypertext in Decision Support Systems. Journal of Management Information Systems, 6(3):119-138, Winter 1989-90.

[Nie90] Jakob Nielsen. HyperText \& HyperMedia. Academic Press, 1990. A very readable introduction to the field.

[Pea91] A. Peake. Brown Brothers Harriman \& co. Personal Communication, March 1991.

[SFS9] P. Darid Stotts and Richard Furuta. Petri net based Hypertext: Document Structure with Browsing Semantics. ACM Transactions on Information Systems, 7(1), January 1989.

[Sim72] H. Simon. Decision and Organization, chapter Thories of Bounded Rationality, pages 162-176. North Holland, Amsterdam, Netherlands, 1972.

[Slo69] P. Slovic. Analyzing the Expert Judge: A Descriptive Study of a Stock Broker's Decision Process. Journal of Applied Psychology, 53:255-263, 1969.

[SLS81] K.L. Stanley, W.G. Lewellen, and G.G Schlarbaum. Further Evidence on the Value of Professional Investment Research. The Journal of Financial Research, 4:1-9, 1981.

[SW88] J.B. Smith and S.F. Weiss. Hypertext. Communications of the ACM, 31(7):816-819, 1988.

[TC76] R.J. Tersine and S.E. Celec. Portfolio management: A practical approach. Atlanta Economic Review, pages 28-33, SeptemberOctober 1976.

[Tom89] Frank Wm. Tompa. A Data Model for Flexible Hypertext Database Systems. ACM Transactions on Information Systems, 7(1), January 1989. 